\title{
A Novel Robust Index to Assess Beat-to-Beat Variability in Heart Rate Time-Series Analysis
}

\author{
Miguel A. García-González*, Member, IEEE, and Ramon Pallàs-Areny, Fellow, IEEE
}

\begin{abstract}
A new index is proposed to estimate the variance of the differentiated heart rate (RR) time series from its truncated histogram. The index is more robust to artifacts than the standard deviation of the differentiated RR time series (rMSDD) and, unlike the pNN50, does not saturate for very high or very low heart rate variability.
\end{abstract}

Index Terms-Artifact rejection, heart rate variability, time-domain analysis.

\section{INTRODUCTION}

$\mathbf{T}$ IME-DOMAIN analysis of heart rate (RR) time series improves the diagnosis and prognosis of some cardiac and neural disorders [1], [2]. Indexes such as the standard deviation of normal-to-normal RR time series quantify heart rate variability (HRV) regardless of its rate of variation. Other indexes quantify fast (beat-to-beat) changes, usually from the differentiated RR (DRR) time series (rMSDD).

The recommended index to analyze DRR variability is the rMSDD [3], which is the standard deviation of the DRR time series (root-mean square of the DRR series) [4]. Although the standard deviation is the best estimate of the variance of a signal [5], the rMSDD index is very sensitive to artifacts. Another common index to characterize DRR variability is the pNN50, which is defined as the percentage of beats whose DRR is larger than $50 \mathrm{~ms}$ [4]. The pNN50 is very robust to artifacts but saturates at $0 \%$ for low HRV and at $100 \%$ for high HRV. The correlation between the rMSDD and pNN50 indexes is very high for normal electrocardiogram (ECG) records [4].

This work compares the rMSDD and pNN50 indexes and proposes a modified rMSDD index with improved performance in front of artifacts that avoids the saturation problems of the pNN50 when analyzing RR time series from healthy subjects and patients.

Manuscript received April 3, 2000; revised July 2, 2000. This work was supported by the Spanish CICYT under Project SAF98-0120. Asterisk indicates corresponding author.

*M. A. García-González is with the Instrumentation and Bioengineering Division (DIB), Department of Electronic Engineering (DEE), Universitat Politècnica de Catalunya (UPC), C/Jordi Girona 1-3, Edifici C-4, 08034 Barcelona, Spain (e-mail: magarcia@eel.upc.es).

R. Pallàs-Areny is with the Instrumentation and Bioengineering Division (DIB), Department of Electronic Engineering (DEE), Universitat Politècnica de Catalunya (UPC), 08034 Barcelona, Spain (e-mail: elerpa@eel.upc.es).

Publisher Item Identifier S 0018-9294(01)04168-4.

\section{ADVANTAGES AND SHORTCOMINGS OF THE rMSDD AND pNN50 INDEXES}

The rMSDD index is very sensitive to artifacts. The DRR time series corresponding to a non contaminated RR time series is

$$
\begin{aligned}
& \mathrm{DRR}_{\text {true }} \\
& =\{\operatorname{RR}(2)-\operatorname{RR}(1), \ldots \operatorname{RR}(n)-\operatorname{RR}(n-1) \\
& \quad \operatorname{RR}(n+1)-\operatorname{RR}(n), \ldots \operatorname{RR}(N)-\operatorname{RR}(N-1)\}
\end{aligned}
$$

where $N$ is the total number of RR intervals recorded.

A false positive (FP) beat replaces one value of the $R_{\text {true }}$ time series by two values. If the FP beat occurs at the $n$th beat we have

$$
\begin{aligned}
\mathrm{RR}_{\mathrm{FP}}= & \{\mathrm{RR}(1) \cdots \mathrm{RR}(n-1), \alpha \mathrm{RR}(n),(1-\alpha) \mathrm{RR}(n), \\
& \operatorname{RR}(n+1) \cdots \operatorname{RR}(N)\}
\end{aligned}
$$

where $0<\alpha<1$ and $1<n<N$. The corresponding DRR time series is

$$
\begin{aligned}
\operatorname{DRR}_{\mathrm{FP}}=\{ & \operatorname{RR}(2)-\mathrm{RR}(1) \cdots \alpha \mathrm{RR}(n)-\mathrm{RR}(n-1), \\
& (1-2 \alpha) \operatorname{RR}(n), \operatorname{RR}(n+1)-(1-\alpha) \operatorname{RR}(n) \\
& \cdots \operatorname{RR}(N)-\operatorname{RR}(N-1)\} .
\end{aligned}
$$

If we designate the rMSDD index of the series $R_{\text {true }}$ by $\mathrm{rMSDD}_{\text {true }}$ and the mean of the series $\mathrm{DRR}_{\text {true }}$ by $\overline{\mathrm{DRR}_{\text {true }}}$, by considering that

$$
\sum_{i=1}^{N-1} \mathrm{DRR}_{\text {true }}(i)=\sum_{i=1}^{N} \mathrm{DRR}_{\mathrm{FP}}(i)=\mathrm{RR}(N)-\mathrm{RR}(1)
$$

we obtain

$$
\overline{\mathrm{DRR}_{\mathrm{FP}}}=\frac{N-1}{N} \overline{\mathrm{DRR}_{\text {true }}} .
$$

On the other hand

$$
\begin{aligned}
\operatorname{rMSDD}_{\mathrm{FP}}^{2} & =\frac{\sum_{i=1}^{N}\left(\mathrm{DRR}_{\mathrm{FP}}(i)-\overline{\mathrm{DRR}_{\mathrm{FP}}}\right)^{2}}{N-1} \\
& =\frac{\left[\sum_{i=1}^{N} \mathrm{DRR}_{\mathrm{FP}}^{2}(i)\right]-N \cdot \overline{\mathrm{DRR}_{\mathrm{FP}}{ }^{2}}}{N-1}
\end{aligned}
$$

but because of the differences between $\mathrm{DRR}_{\text {true }}$ and $\mathrm{DRR}_{\mathrm{FP}}$, we have

$$
\sum_{i=1}^{N} \mathrm{DRR}_{\mathrm{FP}}^{2}(i)
$$




$$
\begin{aligned}
= & {\left[\sum_{i=1}^{N-1} \mathrm{DRR}_{\text {true }}^{2}(i)\right]-[\operatorname{RR}(n)-\operatorname{RR}(n-1)]^{2} } \\
- & {[\operatorname{RR}(n+1)-\operatorname{RR}(n)]^{2}+[\alpha \operatorname{RR}(n)-\operatorname{RR}(n-1)]^{2} } \\
& +(1-2 \alpha)^{2} \operatorname{RR}^{2}(n)+[\operatorname{RR}(n+1)-(1-\alpha) \operatorname{RR}(n)]^{2} .
\end{aligned}
$$

Applying (6) to the $\mathrm{DRR}_{\text {true }}$ series yields

$$
\begin{aligned}
\sum_{i=1}^{N-1} \operatorname{DRR}_{\text {true }}^{2}(i)= & (N-2) \mathrm{rMSDD}_{\text {true }}^{2} \\
& +(N-1) \overline{\mathrm{DRR}}_{\text {true }}
\end{aligned} .
$$

Then, substituting (8) into (7), and (7) and (5) into (6) yields (9), shown at the bottom of the page. Therefore, the error produced by the artifact depends on its amplitude (i.e., its distance to the previous normal beat), which is described by $\alpha$. The closer is $\alpha$ to 0.5 , the larger are the amplitude of the artifact and its effect on the computation of rMSDD. False negative (FN) beats yield similar results.

Because $\mathrm{rMSDD}_{\mathrm{FP}} \neq \mathrm{rMSDD}_{\text {true }}$, artifacts may lead to the wrong diagnostic. One solution is to remove artifacts and then calculate the standard deviation of the artifact-free signal. However, this procedure implies the manipulation of the DRR time series in order to replace several values by approximated artificial values. Another solution is to use an index robust to artifacts and highly correlated with rMSDD $_{\text {true }}$.

The pNN50 is defined as [4]

$$
\operatorname{pNN} 50=\frac{\sum_{i=1}^{N-1} h(i)}{N-1} \cdot 100
$$

where

$$
\begin{cases}h(i)=1 & \text { if }|\mathrm{RR}(i+1)-\mathrm{RR}(i)| \geq 50 \mathrm{~ms} \\ h(i)=0 & \text { if }|\mathrm{RR}(i+1)-\mathrm{RR}(i)|<50 \mathrm{~ms} .\end{cases}
$$

By definition, the Heaviside function $h(i)$ in (11) is $h(i)=1$ for RR interval differences larger than $50 \mathrm{~ms}$ regardless of their amplitude. Consequently, the pNN50 index saturates at $0 \%$ and $100 \%$ when the variance of DRR is respectively very low or very high. Therefore, the pNN50 index does not appropriately describe very low or very high beat-to-beat HRV.

\section{THE rMSDD $_{x}$ INDEX}

Heart rate artifacts always fall inside the tails of the histogram of the DRR time series. Therefore, computing the rMSDD index after excluding the histogram tails should yield an index more robust to artifacts than the common rMSDD and highly correlated to it.

If we define the sDRR time series as the DRR time series sorted from minimum to maximum, sDRR and DRR will have the same histogram. Then we define $\mathrm{sDRR}_{x}$ as the series

$$
\begin{aligned}
\operatorname{sDRR}_{x}=\{ & \operatorname{sDRR}\left(\operatorname{ent}\left[\frac{x N}{100}\right]\right), \operatorname{sDRR}\left(\operatorname{ent}\left[\frac{x N}{100}\right]+1\right) \\
& \left.\ldots, \operatorname{sDRR}\left(N-\operatorname{ent}\left[\frac{x N}{100}\right]\right)\right\}
\end{aligned}
$$

where

$$
\begin{aligned}
& \text { ent }[a] \\
& x
\end{aligned}
$$

$\mathrm{rMSDD}_{x}$ index If $x$ is large enough, $\operatorname{rMSDD}_{x}$ will be very robust to artifacts. Hence, $x$ should be selected according to the estimated fraction of artifacts in the RR time series. Because the estimation of the variance of DRR relies on the standard deviation, $\mathrm{rMSDD}_{x}$ will not saturate as the pNN50 does.

\section{Simulation Studies}

We have compared the linearity and robustness of the pNN50 and $\mathrm{rMSDD}_{x}$ indexes by first analyzing artificial RR time series. The simulated signal for linearity assessment was random Gaussian noise with a mean of $1 \mathrm{~s}$, a length of 300 samples (about $5 \mathrm{~min}$ ) and different standard deviations (SD). For each SD, 100 realizations were computed and the average index calculated. The values for $x$ were zero, one, five, and ten. Fig. 1 shows the dependence of the pNN50 and $\mathrm{rMSDD}_{x}$ on the SD of the input signal. The pNN50 clearly saturates for high SD but the $\mathrm{rMSDD}_{x}$ linearly increases with $\mathrm{SD}$, the slope decreasing for increasing $x$. The correlation between $\mathrm{rMSDD}_{x}$ and $\mathrm{rMSDD}$ after removing the linear trend ranges from 0.985 for $x=1$ to 0.82 for $x=10$.

Since the slope of each straight line in Fig. 1 depends on $x$, we can define a correction factor $(\mathrm{CF})$ as the slope of the rMSDD line divided by that of the line corresponding to $\operatorname{rMSDD}_{x}$. For example, CF is, respectively, $1.0475,1.2477$, and 1.4899 for $x=1,5$, and 10 . The finite length of the series ( 300 samples) has negligible influence on CF. Then, from $\mathrm{rMSDD}_{x}$ we can estimate rMSDD provided that the amplitude distribution of the $\mathrm{RR}$ series is Gaussian.

The robustness of the pNN50 and $\mathrm{rMSDD}_{x}$ indexes to artifacts has been assessed by adding from one to five FP beats. The simulated signals were Gaussian noise with a mean of $1 \mathrm{~s}$, $\mathrm{SD}=30 \mathrm{~ms}$, and 300 samples length. The indexes were calculated for 100 realizations for each artifact number. FP beats were

$$
\operatorname{rMSDD}_{\mathrm{FP}}=\sqrt{\begin{array}{l}
\frac{N-2}{N-1} \mathrm{rMSDD}_{\text {true }}^{2}+\frac{\left(\overline{\mathrm{DRR}_{\text {true }}}\right)^{2}}{N} \\
+\frac{2(1-\alpha) \operatorname{RR}(n) \operatorname{RR}(n-1)-6 \alpha(1-\alpha) \mathrm{RR}^{2}(n)+2 \alpha \operatorname{RR}(n) \operatorname{RR}(n+1)}{N-1}
\end{array}}
$$




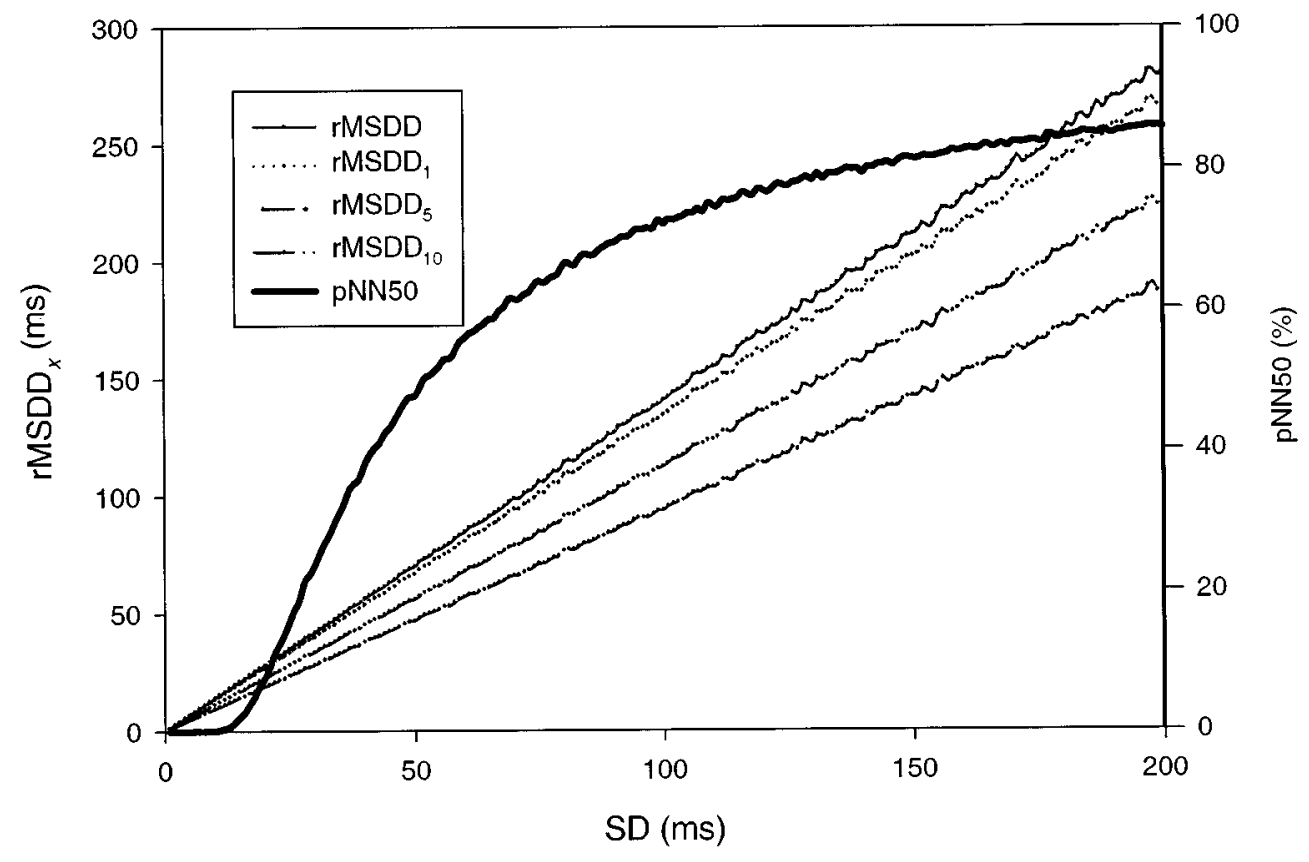

Fig. 1. Dependence of $\mathrm{rMSDD}_{x}$ and $\mathrm{pNN50}$ on the SD of the RR signal.

TABLE I

Sensitivity of Different HRV IndeXes in Front of FAlse Positive Beats Expressed as Relative ERror in Percent (MEAN \pm SD)

\begin{tabular}{c|c|c|c|c|c|c}
\hline \multirow{2}{*}{$\begin{array}{c}\text { Index } \\
\text { without }\end{array}$} & \multicolumn{4}{|c}{ Index error (\%) because of false positives beats } \\
\cline { 3 - 7 } & artifacts & $1 \mathrm{FP}$ & $2 \mathrm{FP}$ & $3 \mathrm{FP}$ & $4 \mathrm{FP}$ & $5 \mathrm{FP}$ \\
\cline { 3 - 7 } & & & & & & \\
\hline pNN50 & $23 \pm 3 \%$ & $3.0 \pm 1.1$ & $6.0 \pm 1.7$ & $9.1 \pm 2.4$ & $12 \pm 3$ & $15 \pm 3$ \\
\hline rMSDD & $42 \pm 2 \mathrm{~ms}$ & $67 \pm 25$ & $116 \pm 28$ & $153 \pm 31$ & $186 \pm 33$ & $218 \pm 33$ \\
\hline rMSDD $_{1}$ & $41 \pm 2 \mathrm{~ms}$ & $0.8 \pm 0.5$ & $4.5 \pm 2.0$ & $14 \pm 6$ & $51 \pm 13$ & $86 \pm 20$ \\
\hline rMSDD $_{5}$ & $34 \pm 2 \mathrm{~ms}$ & $0.6 \pm 0.3$ & $2.1 \pm 0.7$ & $3.9 \pm 1.0$ & $5.8 \pm 1.3$ & $7.7 \pm 1.6$ \\
\hline rMSDD $_{10}$ & $29 \pm 2 \mathrm{~ms}$ & $0.5 \pm 0.3$ & $1.7 \pm 0.6$ & $3.2 \pm 0.9$ & $4.7 \pm 1.2$ & $6.3 \pm 1.4$ \\
\hline
\end{tabular}

introduced by replacing a sample (i.e., a beat) by two samples according to

$$
\left\{\begin{array}{l}
\left\{\operatorname{RR}_{\text {art }}(1) \cdots \mathrm{RR}_{\text {art }}(n-1)\right\} \\
\quad=\{\operatorname{RR}(1) \cdots \operatorname{RR}(n-1)\} \\
\operatorname{RR}_{\text {art }}(n)=\alpha \operatorname{RR}(n) \\
\operatorname{RR}_{\text {art }}(n+1)=(1-\alpha) \operatorname{RR}(n) \\
\left\{\operatorname{RR}_{\text {art }}(n+2) \cdots \operatorname{RR} \text { art }(N+1)\right\} \\
\quad=\{\operatorname{RR}(n+1) \cdots \operatorname{RR}(N)\}
\end{array}\right.
$$

where $\alpha$ was a uniform random variable with $0<\alpha<1$, and the position $n$ was chosen at random.

Table I shows the mean and SD for each index when there are no artifacts, and the relative error (mean $\pm \mathrm{SD}$ ) corresponding to different values for $x$ and number of artifacts, calculated for 100 realizations. The relative error increases with the number of artifacts and decreases for large $x$, as expected. The common rMSDD index has quite a large error. FN (undetected) beats yield similar results.

\section{EXPERIMENTAL RESULTS AND DISCUSSION}

Results from simulated RR series cannot be directly extrapolated to actual RR series because these are not necessarily Gaussian. Therefore, we have tested the correlation between indexes and their robustness to artifacts when calculated for actual ECG recordings.

We have analyzed ambulatory recordings of 18 healthy subjects of the MIT-BIH database [6]. Each RR time series was decomposed in segments that included 300 beats and the corresponding indexes were calculated. Artifacts were identified by variations on DRR greater than 100 ms. Artifacts were so abundant that for most subjects the standard deviation of each index was larger than the mean. Segments severely contaminated by artifacts would probably be rejected in a clinical study, but they have been considered here in order to test the robustness of indexes to artifacts. Table II shows the mean and standard devia- 
TABLE II

AVERAGE AND STANDARD DEVIATION OF THE RELATIVE ERROR OF DifFERENT HRV INDEXES FOR 18 AMBULATORY ECG RECORDINGS FROM THE MIT Database that Have Artifacts. ERrors Are Expressed in Percentage

\begin{tabular}{|c|c|c|c|c|c|}
\hline Recording label & pNN50 & rMSDD & $\mathrm{rMSDD}_{1}$ & $\mathrm{rMSDD}_{5}$ & $\mathrm{rMSDD}_{10}$ \\
\hline 16265 & $9 \pm 36$ & $40 \pm 66$ & $19 \pm 52$ & $2.1 \pm 5.7$ & $1.2 \pm 1.6$ \\
\hline 16272 & $24 \pm 30$ & $161 \pm 197$ & $113 \pm 153$ & $36 \pm 91$ & $11 \pm 37$ \\
\hline 16273 & $2.2 \pm 8.7$ & $18 \pm 45$ & $8 \pm 28$ & $0.5 \pm 1.5$ & $0.3 \pm 0.8$ \\
\hline 16420 & $13 \pm 77$ & $35 \pm 75$ & $16 \pm 49$ & $0.7 \pm 3.8$ & $0.3 \pm 0.7$ \\
\hline 16483 & $10 \pm 24$ & $25 \pm 52$ & $4 \pm 16$ & $0.4 \pm 1.1$ & $0.2 \pm 0.6$ \\
\hline 16539 & $5.9 \pm 9.9$ & $21 \pm 34$ & $13 \pm 23$ & $6.1 \pm 8.9$ & $5.6 \pm 9.6$ \\
\hline 16773 & $1.1 \pm 1.8$ & $4 \pm 11$ & $3.1 \pm 7.4$ & $2.6 \pm 5.2$ & $3.3 \pm 6.8$ \\
\hline 16786 & $1.7 \pm 6.9$ & $10 \pm 34$ & $3 \pm 15$ & $0.4 \pm 1.1$ & $0.3 \pm 0.7$ \\
\hline 16795 & $7.5 \pm 9.6$ & $28 \pm 41$ & $17 \pm 31$ & $9 \pm 16$ & $7.9 \pm 13$ \\
\hline 17052 & $8 \pm 26$ & $43 \pm 77$ & $22 \pm 58$ & $5 \pm 13$ & $3.8 \pm 8.5$ \\
\hline 17453 & $5 \pm 11$ & $39 \pm 65$ & $18 \pm 41$ & $1.1 \pm 2.7$ & $0.7 \pm 1.1$ \\
\hline 18177 & $41 \pm 71$ & $64 \pm 63$ & $36 \pm 51$ & $5 \pm 14$ & $1.9 \pm 2.9$ \\
\hline 18184 & $12 \pm 41$ & $31 \pm 69$ & $10 \pm 40$ & $1.1 \pm 8.9$ & $0.5 \pm 1.7$ \\
\hline 19088 & $3.5 \pm 7.4$ & $22 \pm 37$ & $14 \pm 30$ & $2.1 \pm 6.3$ & $1.5 \pm 5.3$ \\
\hline 19090 & $7 \pm 15$ & $39 \pm 70$ & $14 \pm 36$ & $0.6 \pm 1.1$ & $0.4 \pm 0.7$ \\
\hline 19093 & $2.0 \pm 3.7$ & $13 \pm 24$ & $7 \pm 14$ & $4 \pm 11$ & $2.5 \pm 6.4$ \\
\hline 19140 & $8 \pm 40$ & $17 \pm 45$ & $4 \pm 20$ & $0.7 \pm 1.8$ & $0.5 \pm 1.4$ \\
\hline 19830 & $62 \pm 140$ & $102 \pm 137$ & $48 \pm 97$ & $0.6 \pm 1.1$ & $0.3 \pm 0.7$ \\
\hline
\end{tabular}

tion of the relative error (normalized difference between index before and after artifact removal) for the different indexes and subjects. For the $\operatorname{rMSDD}_{x}$ index, the relative error decreases when $x$ increases, exception made of the record labeled 16773, which has a larger error for $x=10$ than for $x=5$. The error for the rMSDD index is always larger than the error for the $\mathrm{rMSDD}_{1}$ index. The error for the $\mathrm{rMSDD}_{5}$ index is smaller than the error for the pNN50 in 14 from the 18 subjects.

The correlation coefficient between the rMSDD index and the other indexes after artifact removal is higher than 0.95 in most cases. The worst results are those of record 16272 whose correlation coefficients between the rMSDD and the $\mathrm{MSDD}_{1}, \mathrm{rMSDD}_{5}$, and rMSDD $_{10}$ are, respectively, 0.99, 0.86, and 0.72. Kleiger et al. [4] found a good correlation between the pNN50 and the rMSDD for normal subjects. The $\mathrm{MMSD}_{x}$ index introduced here correlates well with the rMSDD index because it is computed from a large fraction of the data values used to compute rMSDD. Because of the linearity between rMSDD and $\mathrm{rMSDD}_{x}, \mathrm{rMSDD}_{\text {true }}$ can be estimated by computing $\operatorname{rMSDD}_{x}$ for an appropriate $x$ and then multiply the result by the corresponding $\mathrm{CF}$, provided that the distribution of the RR time series is known or estimated.

Table II shows that the pNN50 has smaller relative error than the rMSDD index when the ECG record includes artifacts. Nevertheless, records with small pNN50 are more sensitive to artifacts than records with large pNN50. Moreover, the pNN50 saturates when the HRV is small. Fig. 2 shows a RR time series from a healthy subject exercising on a static bicycle and the corresponding pNN50 and rMSDD $_{10}$ obtained by a moving window of 100 beats width. Clearly, the $\mathrm{rMSDD}_{10}$ yields more information than the pNN50.

Patients may have a very low HRV, resulting in a meaningless pNN50 $=0 \%$. Fig. 3, for example, shows the results for the sel103 recording of the MIT-BIH QT database [7]. The $\mathrm{rMSDD}_{10}$ and pNN50 have been calculated in a moving window of 100 beats width. The pNN50 does not provide any information. 


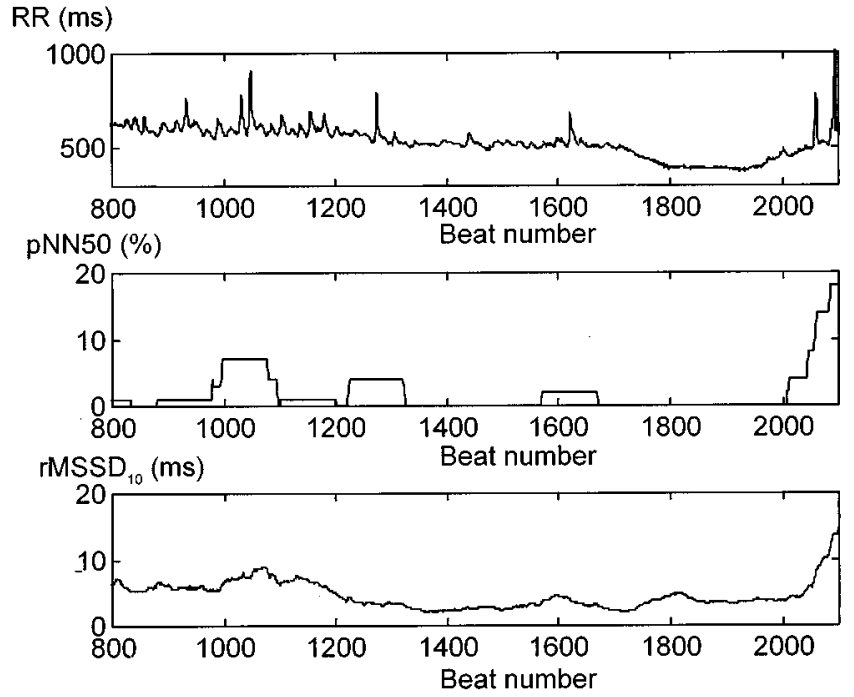

Fig. 2. RR series, pNN50 and $\mathrm{rMSDD}_{10}$ for a healthy subject exercising on a bicycle.

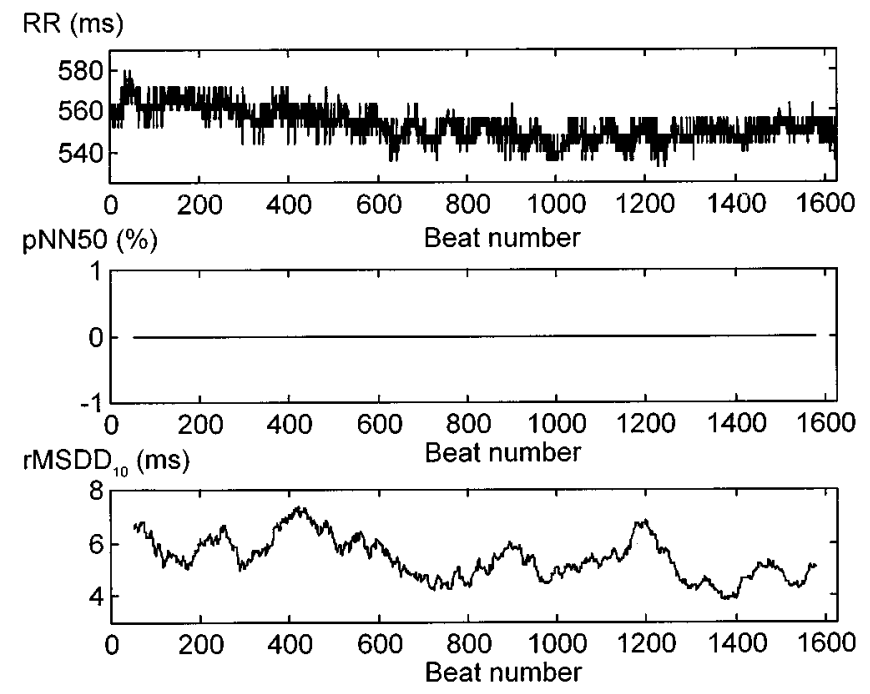

Fig. 3. The pNN50 of an RR series with very low variability saturates to zero, but the rMSDD 10 calculated for a moving window of 100 beats informs about beat-to-beat variability.

\section{CONCLUSION}

The beat-to-beat variability of the rMSDD is often analyzed by the pNN50 and rMSDD indexes [3]. However, the pNN50 tends to saturate for small and large HRV and the rMSDD is very sensitive to artifacts. The proposed $\mathrm{rMSDD}_{x}$ index is very robust to artifacts and does not saturate.

The $\mathrm{rMSDD}_{x}$ index is calculated from the sorted DRR series (series of beat-to-beat RR differences) by excluding the fraction $x$ of samples falling inside the tails of the histogram. Consequently, it correlates very well with the rMSDD index, which is calculated from the complete DRR series. Since the $\mathrm{rMSDD}_{x}$ is linearly related to the rMSDD index (when the RR series is Gaussian) and does not saturate, it is suitable to analyze ECG recordings with very low variability, such as those from some patients, without any artifact removal procedure.

\section{REFERENCES}

[1] M. Di Rienzo, G. Mancia, G. Parati, A. Pedotti, and A. Zanchetti, Eds., Blood Pressure and Heart Rate Variability. Amsterdam, The Netherlands: IOS, 1993.

[2] B. Wennerblom, L. Lurje, H. Tygesen, R. Vahisalo, and A. Hjalmarson, "Patients with uncomplicated coronary artery disease have reduced heart rate variability mainly affecting vagal tone," Heart, vol. 83, pp. 290-294, 2000.

[3] Task Force of the European Society of Cardiology and the North American Society of Pacing and Electrophysiology, "Heart rate variability-Standards of measurement, physiological interpretation, and clinical use," Circulation, vol. 93, pp. 1043-1065, 1996.

[4] R. E. Kleiger, M. S. Bosner, J. N. Rottman, and P. K. Stein, "Time-domain measurements of heart rate variability," J. Ambulatory Monitoring, vol. 6, pp. 256-262, 1987.

[5] J. S. Bendat and A. G. Piersol, Random Data. Analysis and Measurement Procedures, 2nd ed. New York: Wiley, 1986.

[6] G. B. Moody and R. G. Marl, "The MIT-BIH arrhythmia database on CD-ROM and software for use with it," in Computers in Cardiology. Los Alamitos, CA: IEEE Comput. Soc. Press, 1990, pp. 185-188.

[7] P. Laguna, R. G. Mark, A. Goldberger, and G. B. Moody, "A Database for evaluation of algorithms for measurement of QT and other waveform intervals in the ECG," in Computers in Cardiology. Los Alamitos, CA: IEEE Comput. Soc. Press, 1997.

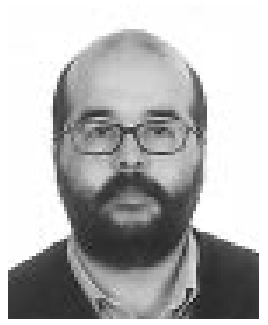

Miguel A. García-González (M’01) received the Ingeniero de Telecomunicación and Doctor Ingeniero Electrónico degrees from the Universitat Politècnica de Catalunya, Barcelona, Spain, in 1993 and 1998, respectively.

$\mathrm{He}$ is a Professor of Electronic Engineering at the same University, and teaches courses in several areas of medical and electronic instrumentation. $\mathrm{He}$ does research on instrumentation methods and ECG and arterial blood pressure measurements. He is interested on time series signal processing and noninvasive measurement of physiological signals.

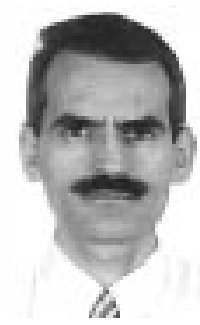

Ramon Pallàs-Areny (M'81-SM'88-F'98) received the Ingeniero Industrial and Doctor Ingeniero Industrial degrees form the Universitat Politècnica de Catalunya, Barcelona, Spain, in 1975 and 1982, respectively.

$\mathrm{He}$ is a Professor of Electronic Engineering at the same University, and teaches courses in several areas of medical and electronic instrumentation. In 1989-1990 he was a Visiting Fulbright Scholar and in 1997-1998 he was an Honorary Fellow at the University of Wisconsin-Madison. He does research on instrumentation methods and sensors based on electrical impedance measurements, sensor interfaces, ECG and arterial blood pressure measurements and electromagnetic compatibility in electronic systems. He is the author of Basic Electronic Instruments (Barcelona, Spain: Marcombo, 1987), Signal Acquisition and Distribution (Barcelona, Spain: Marcombo, 1993), Sensors and Signal Conditioning, 3rd ed. (Barcelona, Spain: Marcombo, 1998), coauthor of An Introduction to Bioengineering (Barcelona, Spain: Marcombo, 1988) and of Electromagnetic Interference in Electronic Systems (Barcelona, Spain: Marcombo, 1991), all published in Spanish He is the author of Basic Error Theory (Barcelona, Spain: Edicions UPC, 1996), coauthor of Interference in Electronic Instruments (Barcelona, Spain: Edicions UPC, 1995), Laboratory of Sensors and Signal Conditioning (Barcelona, Spain: Edicions UPC, 1995), and Sensors and Interfaces, Solved Problems (Barcelona, Spain: Edicions UPC, 1999), all published in Spanish or Catalan. He is also coauthor (with J. G. Webster) of Sensors and Signal Conditioning, 2nd ed. (New York: Wiley, 2001), and Analog Signal Processing (New York: Wiley, 1999).

Dr. Pallàs-Areny was a recipient, with J. G. Webster, of the 1991 Andrew R. Chi Prize Paper Award from the Instrumentation and Measurement Society (IEEE). He is a member of the Biomedical Engineering Society, and the International Society for Measurement and Control. 